\title{
Does Heparin Dosage Calculated with Ideal Body Weight Reduce Blood Product Use in Open Heart Surgery?
}

\author{
Duygu Kara1 ${ }^{1}$, Cafer Mutlu Sarıkaş $^{1}$, Aslı Demir ${ }^{2}$, Mehmet Ali Kayğın $^{3}$, E. Utku Ünal ${ }^{4}$ \\ ${ }^{1}$ Erzurum Regional Training and Research Hospital, Anesthesiology and Reanimation Department, Erzurum \\ ${ }^{2}$ Türkiye Yüksek İhtisas Training and Research Hospital, Anesthesiology and Reanimation Department, Ankara \\ ${ }^{3}$ Erzurum Regional Training and Research Hospital, Cardiovascular Surgery Deparment, Erzurum \\ ${ }^{4}$ Türkiye Yüksek İhtisas Training and Research Hospital, Cardiovascular Surgery Deparment, Ankara
}

\section{Background and Aim}

Heparin is used for its anticoagulant effects and requires neutralization after cardiopulmonary bypass. Anticoagulant efficacy in open heart surgery (OHS) is established with heparin dosages calculated based on patient actual weight. When heparin is administered based on ideal body weight (IBW) rather than actual weight in OHS, changes are observed in activated clotting time (ACT) sufficiency, protamine neutralization dosage, postoperative drainage and blood product transfusion.

\begin{abstract}
Materials and Methods
After ethical committee approval, 100 adults requiring OHS were included.

Patients were divided into two equal groups.

The amount of heparin was calculated from 350 IU/kg based on actual body weight in Group 1 and IBW in Group 2.

ACT was measured after $5 \mathrm{~min}$, and an additional heparin dose was given if ACT $<400$ before cardiopulmonary bypass.
\end{abstract}

Demographic characteristics, intraoperative data, transfusion and heparin-protamine levels were compared between the two groups.

Drainage at postoperative hours 6 and 24, blood transfusion, reoperation, complications, time in intensive care and mortality were also compared.

\section{Results and Discussion}

Mean ages were 59.3 \pm 11.36 in Group 1 and $59.78 \pm 10.34$ in Group 2.

Heparin doses before cardiopulmonary bypass were lower in Group $2(\mathrm{p}<0.001)$.

Intra- and postoperative Erythrocyte and fresh frozen plasma transfusions were higher in Group $1(\mathrm{p}<0.001)$.

Protamine requirement was lower in Group 2 $(\mathrm{p}<0.001)$.

Drainage and length of intensive care stay at postoperative hours 6 and 24 were higher in Group $1(\mathrm{p}<0.001)$.

\section{Conclusion(s)}

Greater use of heparin in OHS can lead to greater postoperative blood/blood product and protamine use and it increases side-effects. In many cases less heparin usage provides enough ACT levels.

In our study, less heparin and protamine were used when administered according to IBW, and complication levels and transfusion requirements were also lower.

\begin{tabular}{|c|c|c|c|c|c|c|c|}
\hline \multicolumn{4}{|c|}{ Table 1. Preoperative characteristics of the stu } & \multicolumn{4}{|c|}{ Table 3. Intra- and postoperative data of the study population } \\
\hline & Group I & Group II & p value & & Group I & Group II & p value \\
\hline & & & & \multicolumn{3}{|l|}{ Surgery type, $n(\%)$} & \multirow[t]{3}{*}{0.34} \\
\hline Age (years) $($ mean \pm SD $)$ & $59.3 \pm 11.3$ & $59.78 \pm 10.34$ & 0.72 & CABG & $19(\% 76)$ & $20(\% 80)$ & \\
\hline Gender; Female/male, n(\%) & $\% 52$ & $\% 76$ & 0.07 & AVR & $1(\% 4)$ & $0(\% 0)$ & \\
\hline Height (cm) & $165.8 \pm 7.4$ & $163.6 \pm 9.7$ & 0.35 & MVR & $3(\% 12)$ & $1(\% 4)$ & \\
\hline $\begin{array}{l}\text { Actual body weight }(\mathbf{k g}) \\
\text { (mean } \pm \text { SD) }\end{array}$ & $74.0 \pm 11.0$ & $73.0 \pm 15.7$ & 0.53 & CABG, MVR & $2(\% 8)$ & $1(\% 4)$ & \\
\hline & - & $59.8 \pm 8.0$ & - & $\begin{array}{l}\text { CABG, AVR } \\
\text { AVR, MVR }\end{array}$ & $\begin{array}{l}0(\% 0) \\
0(\% 0)\end{array}$ & $1(\% 4)$ & \\
\hline$(\operatorname{mean} \pm \mathrm{SD})$ & $9(\% 36)$ & $9(\% 36)$ & 1.00 & $\begin{array}{l}\text { CPB time (sn) } \\
\text { (mean } \pm \text { SD) }\end{array}$ & $77.0 \pm 20.2$ & $83.4 \pm 31.5$ & 0.48 \\
\hline Dyslipidaemia n(\%) & $6(\% 24)$ & $6(\% 24)$ & 1.00 & $\begin{array}{l}\text { Aortic clamp time } \\
(\mathbf{s n})(\text { mean } \pm S D)\end{array}$ & $53.4 \pm 18.4$ & $54.6 \pm 38.7$ & 0.08 \\
\hline Hypertension n(\%) & $13(\% 52)$ & $14(\% 54)$ & \multirow{2}{*}{0.77} & RBC (units) & $3,96 \pm 2.23$ & \multirow[t]{2}{*}{$1.48 \pm 0.51$} & \multirow[t]{2}{*}{$<0.001 *$} \\
\hline Smoker n(\%) & $5(\% 20)$ & $6(\% 24)$ & & $(\operatorname{mean} \pm \mathrm{SD})$ & & & \\
\hline COPD n(\%) & $5(\% 20)$ & $7(\% 28)$ & 0.50 & $\begin{array}{l}\text { Platelets (units) } \\
\text { (mean } \pm \text { SD) }\end{array}$ & 0 & 0 & - \\
\hline OSAS & $0(\% 0)$ & $0(\% 0)$ & - & $\begin{array}{l}\text { FFP (units) } \\
\text { (mean+SD) }\end{array}$ & $2.12 \pm 1.01$ & $1.12 \pm 0.67$ & $<0.001 *$ \\
\hline $\begin{array}{l}\text { Stroke } \\
\text { Renal failure }\end{array}$ & $\begin{array}{l}0(\% 0) \\
0(\%)\end{array}$ & $\begin{array}{l}0(\% 0) \\
0(\% 0)\end{array}$ & - & $\begin{array}{l}\text { Fibrinogen }(180350 \\
\text { mg/dL) }(\text { mean } \pm S D)\end{array}$ & 0 & 0 & - \\
\hline Heart disease & & & 0.64 & $\begin{array}{l}\text { Heparin initial dose } \\
\text { (IU) }(\text { mean } \pm \text { SD) }\end{array}$ & $21856.0 \pm 3505.6$ & $17964.0 \pm 2400.5$ & $<0.001 *$ \\
\hline Ischaemic & $19(\% 76)$ & $20(\% 80)$ & & $\begin{array}{l}\text { Reinjection dose n } \\
(\%)\end{array}$ & $0(\% 0)$ & $6(\% 24)$ & $0.02^{*}$ \\
\hline Valvular & $4(\% 16)$ & $2(\% 8)$ & & $\begin{array}{l}\text { Total heparindose } \\
\text { (IU) (mean } \pm \text { SD) }\end{array}$ & $21856.0 \pm 3505.6$ & $18824.0 \pm 3527.9$ & 0.004* \\
\hline Ischaemic, valvular & $2(\%)$ & $3(\% 12)$ & & $\begin{array}{l}\text { Protamin dose (IU) } \\
(\text { mean } \pm \text { SD) }\end{array}$ & $33500.0 \pm 5199.9$ & $26618.0 \pm 4066.7$ & $<0.001 *$ \\
\hline Antiplatelet agents & & & 0.18 & $\begin{array}{l}\text { ACT-1 (sn) } \\
(\text { mean } \pm \text { SD) }\end{array}$ & $124.3 \pm 20.5$ & $142.6 \pm 20.8$ & $0.005 *$ \\
\hline None & $19(\% 76)$ & $17(\% 68)$ & & $\begin{array}{l}\mathrm{ACT}-2(\mathrm{sn}) \\
(\mathrm{mean} \pm \mathrm{SD})\end{array}$ & $605.2 \pm 20.5$ & $526.7 \pm 74.1$ & $0.002^{*}$ \\
\hline Acetylsalycyclate & $2(\% 8)$ & $7(\% 28)$ & & $\begin{array}{l}\text { ACT-3 }(\mathbf{s n}) \\
(\text { mean } \pm \text { SD })\end{array}$ & $117.5 \pm 14.8$ & $127.8 \pm 12.4$ & 0.001* \\
\hline Warfarin & $1(\% 4)$ & $0(\%)$ & & $\begin{array}{l}\text { Blood loss - H6 } \\
(\mathrm{mL})(\mathrm{mean} \pm \mathrm{SD})\end{array}$ & $548.0 \pm 108.4$ & $403.2 \pm 101.2$ & $<0.001 *$ \\
\hline Clopidogrel & $3(\% 12)$ & $1(\% 4)$ & & $\begin{array}{l}\text { Blood loss- H24 } \\
(\mathrm{mL})(\text { mean } \pm \mathrm{SD})\end{array}$ & $770.0 \pm 170.7$ & $596.4 \pm 149.6$ & $<0.001 *$ \\
\hline & & & & $\begin{array}{l}\text { Post-op RBC (units) } \\
\text { (mean } \pm \text { SD) }\end{array}$ & $2.3 \pm 0.9$ & $1.56 \pm 0.5$ & $0.001 *$ \\
\hline $\begin{array}{l}\text { SD: standart deviation, COPD: cl } \\
\text { OSAS: obstructive sleep apnoea s }\end{array}$ & $\begin{array}{l}\text { ronic obstruct } \\
\text { yndrome }\end{array}$ & ctive pulmonary & disease, & $\begin{array}{l}\text { Post-op platelet } \\
\text { (units) (mean } \pm \text { SD) }\end{array}$ & 0 & 0 & - \\
\hline Table 2. Laboratory data of th & study popu & ulation(mean: & \pm SD) & $\begin{array}{l}\text { Post-op FFP (units) } \\
(\text { mean } \pm \text { SD })\end{array}$ & $2.3 \pm 0.6$ & $1.0 \pm 0.7$ & $<0.001 *$ \\
\hline & & & & Reoperation n(\%) & $3(\% 12)$ & $2(\% 8)$ & 1.00 \\
\hline & Group I & Group II & $\begin{array}{c}\mathbf{p} \\
\text { value }\end{array}$ & Complication & & & 0.35 \\
\hline Haemoglobin (gr/dL) & $14.2 \pm 1.6$ & $14.9 \pm 1.3$ & 0.07 & None & $19(\% 76)$ & $21(\% 84)$ & \\
\hline Haematocrit (\%) & $42.2 \pm 6.0$ & $45.1 \pm 4.1$ & 0.10 & Stroke & $2(\% 8)$ & $0(0)$ & \\
\hline Platelets $\left(10^{9} \mathrm{~L}^{-1}\right)$ & $239,7 \pm 68$ & $265,4 \pm 64$ & 0.18 & Sepsis & $2(\% 8)$ & $1(\% 4)$ & \\
\hline Phrothrombin time (10.5-14.9 & $13.2 \pm 1.58$ & $12.7 \pm 0.8$ & 0.28 & Acute renal failure & $1(\% 4)$ & $0(\% 0)$ & \\
\hline $\begin{array}{l}\text { sn) } \\
\text { Aptt (21-35sn) }\end{array}$ & $25.8 \pm 5.0$ & $25.0 \pm 2.3$ & 0.79 & $\begin{array}{l}\text { Respiratuar } \\
\text { complication }\end{array}$ & $1(\% 4)$ & $3(\% 12)$ & \\
\hline Fibrinogen (180-350 mg/dL) & $\begin{array}{c}353.0 \pm 48 . \\
4\end{array}$ & $\begin{array}{c}326.1 \pm 73 \\
8\end{array}$ & 0.11 & $\begin{array}{l}\text { Length of ICU stay } \\
\text { (days) (mean } \pm S D)\end{array}$ & $3.8 \pm 1.2$ & $3.0 \pm 0.9$ & $0.005 *$ \\
\hline SD: standart deviation, aPTT: ac & tivatedpartia & althromboplast & tin time & Mortality n(\%) & $5(\% 20)$ & $2(\% 8)$ & 0.41 \\
\hline & & & & $\begin{array}{l}* \text { : statisticallysignifice } \\
\text { mean_SEM, or n (\%). } \\
\text { hourpostoperation; } \mathrm{H} 6\end{array}$ & $\begin{array}{l}\text { nt, SD: standart de } \\
\text { FFPr6, freshfrozen } \\
\text { sixthhourpostoper }\end{array}$ & $\begin{array}{l}\text { iation, Valuesaree } \\
\text { lasma; H24, 24th } \\
\text { tion; }\end{array}$ & essed as \\
\hline
\end{tabular}

